\title{
Solving fuzzy differential equations using implicit multistep block method
}

\begin{abstract}
In this paper, an implicit multistep block method of order three is proposed for solving fuzzy differential equations. The method is based on diagonally implicit multistep block method where the coefficients of the lower triangular matrix entries are zero. This method works by moving two steps in a block, thus the values of $y n+1$ and $y n+2$ are approximated simultaneously. The formulas are derived by using Lagrange interpolating polynomial. The method is carried out by combining the predictor and corrector formulas. The performances of the block method are compared with the existing methods by solving some numerical problems.
\end{abstract}

Keyword: Block method; Fuzzy differential equations; Lower triangular matrix 